\title{
FREE $S^{3}$-ACTIONS ON 2-CONNECTED NINE-MANIFOLDS(') BY
}

RICHARD I. RESCH

\begin{abstract}
ABSTRACr. In this paper a classification of free $S^{3}$-actions on 2-connected 9-manifolds is obtained by examining the corresponding principal $S^{3}$-bundles. The orbit spaces that may occur are determined and it is proved that there are exactly two homotopy classes of maps from each of these spaces into the classifying space for principal $S^{3}$-bundles. It is shown that the total spaces of the corresponding bundles are distinct, yielding the main result that for each nonnegative integer $k$, there exist exactly two 2-connected 9-manifolds which admit free $S^{3}$-actions and, furthermore, the actions on each of these manifolds are unique.
\end{abstract}

1. Introduction. In [4] free $S^{3}$-actions on simply connected 8-manifolds with torsion-free homology were classified and it was proved, without restriction on the homology groups, that if a simply connected 8-manifold admits a free $S^{3}$-action, then that action is unique. It is the purpose of this paper to present similar results for free $S^{3}$-actions on 2-connected 9-manifolds.

In $\$ 2$ it is shown that the orbit spaces of such an action must be the connected sum of $S^{6}$ with $k$ copies of $S^{3} \times S^{3}$ for some nonnegative integer $k$. These spaces will be denoted by $N_{k}$. The Puppe sequence is then used to prove the following theorem:

THEOREM 2. For each nonnegative integer $k$, there are at most two distinct free $S^{3}$-actions on 2-connected 9-manifolds with third homology group free of rank $2 k+1$.

In $\$ \S 3$ and 4 it is shown that this result can be sharpened by first determining that for each $k$, there are exactly two principal $S^{3}$-bundles over $N_{k}$ and then that the total spaces of these bundles are homotopically distinct. These results yield the main theorem:

THEOREM 3. For each nonnegative integer $k$, there exist exactly two 2-connected 9-manifolds with third homology group free of rank $2 k+1$ which admit free $S^{3}$-actions. These manifolds are $S^{3} \times N_{k}$ and the nontrivial $S^{3}$-bundle over

Presented to the Society, January 16, 1974; received by the editors June 15, 1975.

AMS (MOS) subject classifications (1970). Primary 57E25; Secondary 55F15.

$K e y$ words and phrases. Free $S^{3}$-action, principal $S^{3}$-bundle, Puppe sequence.

(') This paper is an extension of part of the author's Ph.D. dissertation prepared at the University of Connecticut under the direction of Professor Soon-Kyu Kim.

- American Mathematical Society 1977 
$N_{k}$ and, furthermore, there is a unique free $S^{3}$-action on each of these manifolds.

2. Preliminary results. Let $M$ denote a closed 2-connected differentiable 9-manifold which admits a free differentiable $S^{3}$-action with orbit space $N$. Then $N$ is a 2-connected 6-manifold which, according to Smale [6], is diffeomorphic to the connected sum of $S^{6}$ with $k$ copies of $S^{3} \times S^{3}$ for some nonnegative integer $k$. We denote these manifolds by $N_{k}$.

The integral homology groups of $N_{k}$ are easily seen to be $Z$ in dimensions zero and six and the direct sum of $2 k$ copies of $Z$ in dimension three. Thus using the Gysin homology sequence of the principal $S^{3}$-bundle $S^{3} \rightarrow M \rightarrow N_{k}$ and Poincare duality we can calculate the homology groups of $M$. We state the result in the following theorem:

THEOREM 1. If the 2-connected 9-manifold $M$ admits a free differentiable $S^{3}$-action with orbit space $N_{k}$, then its homology must be torsion-free. In particular, $H_{0}(M) \simeq H_{9}(M) \simeq Z, H_{3}(M) \simeq H_{6}(M) \simeq \bigoplus_{2 k+1} Z$, and $H_{i}(M) \approx 0$ for $i \neq 0,3,6,9$.

It follows from Theorem 1 that all free $S^{3}$-actions on a given 2-connected 9-manifold $M$ must have the same orbit space $N$, since $H_{3}(M) \simeq \bigoplus_{2 k+1} Z$ if and only if $H_{3}(N) \simeq \bigoplus_{2 k} Z$. We will now show that each of the possible orbit spaces $N_{k}$ can be the base space of at most two principal $S^{3}$-bundles.

THEOREM 2. For each nonnegative integer $k$, there are at most two distinct free $S^{3}$-actions on 2-connected 9-manifolds with third homology group free of rank $2 k+1$.

Proof. To calculate the number of principal $S^{3}$-bundles over $N_{k}$, we compute homotopy classes of maps of $N_{k}$ into $H P^{\infty}$, the classifying space for principal $S^{3}$-bundles. Since the dimension of $N_{k}$ is $6,\left[N_{k}, H P^{\infty}\right]=\left[N_{k}\right.$, $\left.H P^{1}\right]=\left[N_{k}, S^{4}\right]$, where the square brackets denote homotopy classes of maps. Consider the following exact sequence obtained from the Puppe sequence

$$
\left[N_{k}^{3}, S^{4}\right] \leftarrow\left[N_{k}^{6}, S^{4}\right] \stackrel{f}{\leftarrow}\left[N_{k}^{6} / N_{k}^{3}, S^{4}\right] \leftarrow \ldots
$$

where $N_{k}^{i}$ is the $i$-skeleton of $N_{k}$. Note that $N_{k}^{3}$ is a bouquet of 3-spheres so $\left[N_{k}^{3}, S^{4}\right]=0$ and $f$ is onto. On the other hand, $N_{k}^{6} / N_{k}^{3}=S^{6}$ and $\left[S^{6}\right.$, $\left.S^{4}\right] \simeq \pi_{6}\left(S^{4}\right) \simeq Z_{2}$. Thus $\left[N_{k}^{6}, S^{4}\right]$ can have at most two elements and we are done.

3. Determination of $\left[N_{k}, S^{4}\right]$. In this section we prove that there are two principal $S^{3}$-bundles over each $N_{k}$ by proving that $\left[N_{k}, S^{4}\right] \simeq Z_{2}$. For $k=0$ we have $N_{0}=S^{6}$ and, thus, $\left[N_{0}, S^{4}\right] \simeq \pi_{6}\left(S^{4}\right) \simeq Z_{2}$. We state this result for completeness. 
LEMMA $1 .\left[N_{0}, S^{4}\right] \simeq Z_{2}$.

The result for $k=1, N_{1}=S^{3} \times S^{3}$, is proved in [5]. There the Puppe sequence of the map $f: S^{5} \rightarrow S^{3} \vee S^{3}$, representing the Whitehead product by which a six-cell is attached to $S^{3} \vee S^{3}$ to obtain $S^{3} \times S^{3}$, is used to prove that $\left[S^{3} \times S^{3}, S^{4}\right] \simeq Z_{2}$. We now generalize this idea to prove the following lemma:

LEMMA 2. $\left[N_{k}, S^{4}\right]=Z_{2}$ for $k \geqslant 1$.

Proof. In this proof we will use $N$ to denote any of the orbit spaces $N_{k}$ for $k \geqslant 1$. Let $\left\{N, S^{4}\right\}_{0}=\lim \left[\Sigma^{n} N, S^{n+4}\right]$ where $\Sigma^{n} X$ is the $n$th suspension of $X$ and lim is the inverse limit. We will first show that $\left[N, S^{4}\right]$ is isomorphic to $\left\{N, S^{4}\right\}_{0}$ and then that the latter is $Z_{2}$.

Let $\Omega^{n}$ represent the $n$th loop space and $Q\left(S^{4}\right)=\lim \Omega^{n} S^{n+4}$. Then

$$
\pi_{4}\left(\Omega^{n} S^{n+4}\right) \simeq\left[S^{4}, \Omega^{n} S^{n+4}\right] \simeq\left[\Sigma^{n} S^{4}, S^{n+4}\right] \simeq\left[S^{n+4}, S^{n+4}\right] \simeq Z \text {. }
$$

Let $i \in Z$ represent a map $\sigma: S^{4} \rightarrow \Omega^{n} S^{n+4}$. Taking the limit we have the usual inclusion $I: S^{4} \rightarrow Q\left(S^{4}\right)$. Now the cofibration $F \rightarrow Q\left(S^{4}\right) \rightarrow^{p}$ $Q\left(S^{4}\right) / S^{4}$ can be expanded as a fiber mapping sequence [3, p. 138] into the following diagram where the lifting $J: S^{4} \rightarrow F$ exists since the composition $p I$ is nullhomotopic:

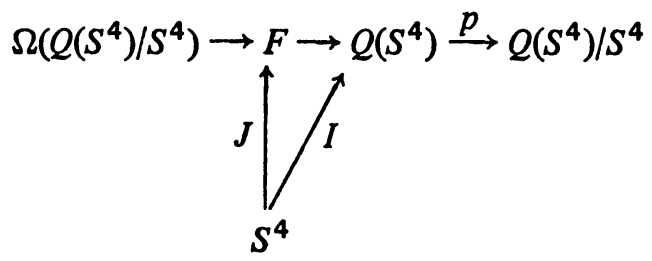

Now by the EHP sequence [2] $\pi_{i}\left(Q\left(S^{4}\right) / S^{4}\right)=0$ for $i \leqslant 7$ and $J_{\#}: \pi_{i}\left(S^{4}\right) \rightarrow$ $\pi_{i}(F)$ is an isomorphism for $i<10$. Therefore, if we consider homotopy classes of maps of $N$ into each term of this diagram we obtain the following:

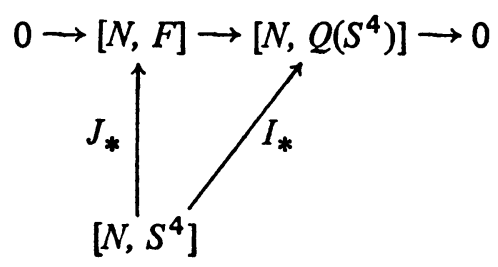

The zero terms are the result of the 7- and 6-connectedness of $Q\left(S^{4}\right) / S^{4}$ and its loop space, respectively. Since $J_{*}$ is an isomorphism and the diagram commutes, $I_{*}$ must also be an isomorphism.

If $\Sigma^{n} f$ is the $n$th suspension of a map $f: N \rightarrow S^{4}$, then $I_{*}(f)=\lim \Sigma^{n} f$. Thus, $I_{*}$ is an isomorphism from $\left[N, S^{4}\right]$ to $\left\{N, S^{4}\right\}_{0}=\lim \left[\Sigma^{n} N, S^{n+4}\right]$. 
To see that $\left\{N, S^{4}\right\}_{0}=Z_{2}$ consider the cofibering $N^{5} \rightarrow N^{6} \rightarrow N^{6} / N^{5}$ where $N^{i}$ is the $i$-skeleton of $N$. Thus, $N^{5}$ is homotopically a wedge of 3-spheres (recall that $N$ is a connected sum of copies of $S^{3} \times S^{3}$ ). Therefore, $N^{6} / N^{5}$ is a homotopy 6-sphere. Considering homotopy classes of maps of each space in this cofibering into $S^{4}$ and taking limits yields the following exact sequence:

$$
\begin{aligned}
\left\{S^{6}, S^{4}\right\}_{-1} & \rightarrow\left\{N^{6}, S^{4}\right\}_{-1} \stackrel{f}{\rightarrow}\left\{N^{5}, S^{4}\right\}_{-1} \\
& \stackrel{\delta}{\rightarrow}\left\{S^{6}, S^{4}\right\}_{0} \rightarrow\left\{N^{6}, S^{4}\right\}_{0} \rightarrow\left\{N^{5}, S^{4}\right\}_{0} .
\end{aligned}
$$

Here $\left\{X, S^{4}\right\}_{-1}$ means $\left\{\Sigma X, S^{4}\right\}_{0}$. Now $\left\{N^{5}, S^{4}\right\}_{0} \simeq 0$ since it is obtained by suspension from $\left[N^{5}, S^{4}\right]$ which is trivial. Similarly, $\left\{S^{6}, S^{4}\right\}_{0}=$ $Z_{2}$ since it is obtained from $\left[S^{6}, S^{4}\right]$ which is $Z_{2}$.

Since $\Sigma N^{5}=S^{4} \vee \cdots \vee S^{4}$ and $\Sigma N^{6}=S^{7} \vee S^{4} \vee \cdots \vee S^{4}, \Sigma N^{5}$ is a retract of $\Sigma N^{6}$ and $f$ has an inverse. Therefore, $\delta$ is 0 , and since

$$
\left\{N^{5}, S^{4}\right\}_{0}=0
$$

it follows that $\left\{N^{6}, S^{4}\right\}_{0} \simeq\left[N^{6}, S^{4}\right] \simeq Z_{2}$ and the proof is complete.

Lemmas 1 and 2 imply that for each possible orbit space there are exactly two free $S^{3}$-actions on 2-connected 9-manifolds.

4. Distinguishing between the total spaces. In this section we prove that the total spaces of the trivial and nontrivial principal $S^{3}$-bundles over $N_{k}, k>0$, are homotopically distinct. First, recall that $N_{0}=S^{6}$ and let $E$ denote the total space of the nontrivial $S^{3}$-bundle over $S^{6}$.

LEMMA 3. $E$ and $S^{3} \times S^{6}$ are not of the same homotopy type.

Proof. Consider the following commutative diagram where the bottom row is the homotopy sequence of the trivial bundle, the vertical arrows are the Hurewicz homomorphisms and the top isomorphism is induced by $p: S^{3} \times$ $S^{3} \rightarrow S^{6}$ in the Gysin sequence:

$$
\begin{aligned}
& H_{6}\left(S^{3} \times S^{6}\right) \stackrel{\simeq}{\longrightarrow} H_{6}\left(S^{6}\right) \\
& \prod_{\pi_{6}\left(S^{3} \times S^{6}\right) \stackrel{p_{\#}}{\longrightarrow} \pi_{6}\left(S^{6}\right) \stackrel{\Delta}{\longrightarrow} \pi_{5}\left(S^{3}\right) \simeq z_{2}}
\end{aligned}
$$

From the homotopy sequence of the trivial bundle we have that $\Delta=0$. Hence, $p_{\#}$ must be onto. Since the diagram commutes, $\alpha$ must also be onto.

If $S^{3} \times S^{6}$ is replaced in the diagram by $E$, then $\Delta$ is onto. Thus, by the commutativity and the exactness of the bottom row $p_{\#}$ and $\alpha$ are not onto. Therefore, the Hurewicz map $\alpha$ from $\pi_{6}$ to $H_{6}$ differs for $S^{3} \times S^{6}$ and $E$. 
Since $\alpha$ distinguished homotopy types these spaces must be homotopically distinct.

It is proved in [5] that the trivial and nontrivial $S^{3}$-bundles over $S^{3} \times S^{3}$ are distinct. The method used is to assume that the total spaces of these bundles are homotopically equivalent and to construct a map of a 6-sphere into $E$, the nontrivial bundle over $S^{6}$, representing a generator of the sixth homology group of $E$. This contradicts the proof of Lemma 3, however, since it was shown there that the Hurewicz map from $\pi_{6}(E)$ to $H_{6}(E)$ is not onto.

The following lemma using secondary cohomology operations establishes the result in general for the orbit spaces $N_{k}, k \geqslant 1$.

LEMMA 4. For each integer $k \geqslant 1$, the trivial and nontrivial principal $S^{3}$-bundles over $N_{k}$ are homotopically distinct.

Proof. Let $\Phi$ be the secondary cohomology operation based on the Adem relation $S q^{3} S q^{1}+S q^{2} S q^{2}=0$ as in [3, p. 163]. $\Phi$ is defined with zero indeterminacy on $H^{3}$ of the total spaces of both the trivial and nontrivial $S^{3}$-bundles over $N_{k}$. Since the trivial bundle is a product, $\Phi$ is zero in that case.

The 6-skeleton of the nontrivial bundle can be decomposed as follows:

$$
S_{1}^{3} \vee \cdots \vee S_{2 k+1}^{3} \cup e_{\left.i_{1}, i_{2}\right]}^{6} \cup \cdots \cup e_{\left[i_{1}, i_{2 k+1}\right]}^{6} \cup e_{\theta}^{6}
$$

where $\theta=\left[i_{2}, i_{3}\right]+\left[i_{4}, i_{5}\right]+\cdots+\left[i_{2 k}, i_{2 k+1}\right]+\left(\eta_{1}\right)^{2}, i_{n} \in \pi_{3}\left(S_{n}^{3}\right), \eta_{1} \in$ $\pi_{3}\left(S_{1}^{3}\right)$ and each $\left[i_{1}, i_{m}\right]$ is the Whitehead product for attaching the six-cell $e^{6}$. $\Phi$ detects the $\left(\eta_{1}\right)^{2}$ component of $\theta$, that is, it is nonzero on $H^{3}\left(S_{1}^{3} \cup e_{\theta}^{6} ; Z_{2}\right)$. This, $\Phi$ distinguishes between the trivial and nontrivial $S^{3}$-bundles over $N_{k}$.

Lemmas 3 and 4 together complete our classification of free $S^{3}$-actions on 9-manifolds by yielding the following theorem:

THEOREM 3. For each nonnegative integer $k$, there exist exactly two 2-connected 9-manifolds with third homology group free of rank $2 k+1$ which admit free $S^{3}$-actions. These manifolds are $S^{3} \times N_{k}$ and the nontrivial $S^{3}$-bundle over $N_{k}$ and, furthermore, there is a unique free $S^{3}$-action on each of these manifolds.

\section{REFERENCES}

1. G. E. Bredon, Introduction to compact transformation groups, Academic Press, New York and London, 1972.

2. R. J. Milgram, Unstable homotopy from the stable point of view, Lecture Notes in Math., vol. 368, Springer-Verlag, Berlin, 1974. MR 50 \# 1235.

3. R. E. Mosher and M. C. Tangora, Cohomology operations and applications in homotopy 
theory, Harper and Row, London and New York, 1968. MR 37 \#2223.

4. R. I. Resch, Free $S^{3}$-actions on simply connected eight-manifolds, Proc. Amer. Math. Soc. 49 (1975), 461-468.

5. , The classification of free differentiable $S^{3}$-actions on eight-and nine-dimensional manifolds, Ph. D. thesis, Univ. of Connecticut, 1973.

6. S. Smale, On the structure of manifolds, Amer. J. Math. 84 (1962), 387-399. MR 27 \#2991.

7. N. E. Steenrod, The topology of fibre bundles, Princeton Univ. Press, Princeton, N. J., 1951. MR 12, 522.

Division of Science and Mathematics, The College of the Virgin Islands, St. Thomas, U. S. VIRGIN ISLANDS 00801 